\title{
Association between dietary phylloquinone intake and peripheral metabolic risk markers related to insulin resistance and diabetes in elderly subjects at high cardiovascular risk
}

\author{
Martí Juanola-Falgarona ${ }^{1,2,3}$, Jordi Salas-Salvadón ${ }^{1,2,3^{*}}$, Ramon Estruch ${ }^{2,3,4}$, Maria P Portillo ${ }^{3,5}$, Rosa Casas ${ }^{2,3,4}$,
} Jonatan Miranda ${ }^{3,5}$, Miguel A Martínez-González $z^{3,6}$ and Mònica Bulló $1,2,3^{*}$

\begin{abstract}
Background: Vitamin $\mathrm{K}$ has been related to glucose metabolism, insulin sensitivity and diabetes. Because inflammation underlies all these metabolic conditions, it is plausible that the potential role of vitamin K in glucose metabolism occurs through the modulation of cytokines and related molecules. The purpose of the study was to assess the associations between dietary intake of vitamin $\mathrm{K}$ and peripheral adipokines and other metabolic risk markers related to insulin resistance and type 2 diabetes mellitus.
\end{abstract}

Methods: Cross-sectional and longitudinal assessments of these associations in 510 elderly participants recruited in the PREDIMED centers of Reus and Barcelona (Spain). We determined 1-year changes in dietary phylloquinone intake estimated by food frequency questionnaires, serum inflammatory cytokines and other metabolic risk markers.

Results: In the cross-sectional analysis at baseline no significant associations were found between dietary phylloquinone intake and the rest of metabolic risk markers evaluated, with exception of a negative association with plasminogen activator inhibitor-1. After 1-year of follow-up, subjects in the upper tertile of changes in dietary phylloquinone intake showed a greater reduction in ghrelin $(-15.0 \%)$, glucose-dependent insulinotropic peptide $(-12.9 \%)$, glucagon-like peptide-1 (-17.6\%), IL-6 (-27.9\%), leptin (-10.3\%), TNF $(-26.9 \%)$ and visfatin $(-24.9 \%)$ plasma concentrations than those in the lowest tertile (all $p<0.05$ ).

Conclusion: These results show that dietary phylloquinone intake is associated with an improvement of cytokines and other markers related to insulin resistance and diabetes, thus extending the potential protection by dietary phylloquinone on chronic inflammatory diseases.

Trial registration: http://www.controlled-trials.com as ISRCTN35739639

Keywords: Vitamin K, Inflammation, Insulin resistance, Diabetes

\footnotetext{
*Correspondence: jordi.salas@urv.cat; monica.bullo@urv.cat

'Human Nutrition Unit, Faculty of Medicine and Health Sciences, IISPV,

Universitat Rovira i Virgili, C/Sant Llorenç 21, 43201, Reus, Spain

${ }^{2} \mathrm{ClBER}$ obn Physiopathology of Obesity and Nutrition, Institute of Health

Carlos III (ISCIII), Madrid, Spain

Full list of author information is available at the end of the article
} 


\section{Introduction}

Vitamin $\mathrm{K}\left(\mathrm{K}_{1}\right.$ or phylloquinone and $\mathrm{K} 2$ or menaquinones) is recognized as an essential element in the synthesis of carboxylate clotting factors involved in prothrombotic disorders and cardiovascular disease. More recently, it has been reported that vitamin $\mathrm{K}$ also participates in the gamma-carboxylation reactions of other proteins such as osteocalcin, and may also exert a protective role against age-related bone loss [1,2]. However, additional roles of vitamin $\mathrm{K}$, independent of these effects have been described [3]. Thus, there is evidence that both osteocalcin and vitamin $\mathrm{K}$ may have a potential beneficial role in glucose metabolism, insulin sensitivity and type 2 diabetes (T2DMs) [4-7]. Since inflammation underlies all these chronic metabolic conditions, it is plausible that the potential role of vitamin $\mathrm{K}$ in glucose metabolism partly occurs through the modulation of cytokines and other metabolic risk markers related to insulin resistance and diabetes.

In-vitro studies have shown an anti-inflammatory effect of vitamin K. Human macrophage THP-1 cells incubated with vitamin $\mathrm{K}$ reduced the interleukin-6 (IL-6) expression compared to non-incubated cells. Likewise, rats fed with a vitamin K-deficient diet showed an enhanced expression of genes involved in the acute inflammatory response [8]. In a subsample of 1,321 subjects from the Framingham Offspring Study, both plasma phylloquinone and dietary phylloquinone intake were inversely associated with peripheral concentrations of some inflammatory markers [9]. However, in a 3-year randomized clinical trial designed to assess the effect of vitamin $\mathrm{K}$ supplementation on bone loss, no differences were found in the plasma IL-6, Creactive protein or osteoprotegerin concentrations of participants receiving or not a phylloquinone supplement [10].

The purposes of the present study were to assess the cross-sectional associations between dietary intake of vitamin $\mathrm{K} 1$ and selected adipokines or other metabolic risk markers related to inflammation, insulin resistance and diabetes; and to longitudinally analyse the associations between changes in dietary phylloquinone intake and changes in these risk markers after one-year of follow-up in a cohort of elderly subjects at high cardiovascular risk.

\section{Methods}

\section{Study population}

In the present study we conducted a cross-sectional and a longitudinal assessment of 568 consecutively recruited participants for the PREDIMED trial centers of Reus and Barcelona (Spain). The PREDIMED study is a large, parallel group, multicenter, controlled, randomized, clinical trial designed to evaluate the effect of the Mediterranean diet on the primary prevention of cardiovascular disease in elderly. Participants were community-dwelling men and women aged $55-80$ and $60-80$ years, respectively.
At baseline they were free of cardiovascular disease and were either diabetic or met at least three or more coronary heart disease risk factors including smoking, hypertension (blood pressure $\geq 140 / 90 \mathrm{mmHg}$ or treatment with antihypertensive drugs), dyslipidemia [low-density lipoprotein cholesterol level $\geq 160 \mathrm{mg} / \mathrm{dL}$ or treatment with hypolipidemic drugs], high-density lipoprotein cholesterol level of $40 \mathrm{mg} / \mathrm{dL}$ or lower, overweight [Body mass index $\geq 25 \mathrm{~kg} / \mathrm{m}^{2}$ ] or family history of premature cardiovascular disease. Exclusion criteria included any severe chronic illness, drug or alcohol addiction, history of allergy or intolerance to olive oil or nuts, or a low predicted likelihood of changing dietary habits according to Prochaska and DiClemente's stages-of-change model. The participants included in the PREDIMED study were randomly assigned to 3 intervention groups: a Mediterranean Diet with virgin olive oil, a Mediterranean Diet with mixed nuts and a control group where a low-fat diet is recommended according to the American Heart Association guidelines. Full details of the PREDIMED study protocol have been published elsewhere [11,12]. The study protocol was approved by the institutional review boards of Hospital Clínic and Hospital Universitari Sant Joan de Reus, and all subjects agreed to participate in the study and gave their written informed consent. The trail was registered in http://www.controlled-trials. com as ISRCTN35739639.

\section{Dietary assessment}

Two individual motivational interviews every 3 months to negotiate nutrition goals, and group educational sessions on a quarterly basis, focused to adapt the customary diet to a traditional Mediterranean diet, were compared with a control group, which received verbal instructions and a leaflet recommending the National Cholesterol Education Program Adult Treatment Panel III dietary guidelines (www.predimed.org). At baseline and after one-year of follow-up participants were assessed by trained dieticians who administered a previously validated 137-item food frequency questionnaire (FFQ) [13]. Additionally, a validated brief 14-item Mediterranean Diet Adherence Screener was used to assess adherence to the traditional Mediterranean Diet (MedDiet) where subjects were asked for their consumption of the most common Mediterranean foods [14]. Subjects with a higher consumption of healthier foods such as olive oil, vegetables, legumes, fruit, nuts, fish and seafood, white meat instead of red meat, sofrito and red wine scored higher in this questionnaire. Energy and nutrient intakes were calculated from Spanish food composition tables $[15,16]$. Dietary phylloquinone intake was calculated using the database of the US Department of Agriculture, Human Nutrition Research Center on Aging at Tufts University (http://www.nal.usda.gov/ fnic/foodcomp/search) and the reproducibility and relative 
validity of a self-administered FFQ used in the study was validated for dietary phylloquinone intake. Reproducibility for dietary phylloquinone intake explored by the Pearson correlation coefficient $(r)$ ranged was 0.755 , and the intraclass correlation coefficient (ICC) was $0.860, \mathrm{p}<0.001$.

\section{Other measurements}

Additional information was collected on subjects' medical record, including the use of medication. Trained personnel measured baseline weight, height and waist circumference as previously reported $[11,12]$, as well as blood pressure in triplicate with a validated semiautomatic oscillometer (Omron HEM-705CP, Hoofddorp, the Netherlands). Leisure-time physical activity was evaluated using the validated Spanish version of the Minnesota leisure-time physical activity questionnaire. Centralized laboratory biochemical analyses were performed on blood samples obtained in fasting conditions. Plasma glucose, serum cholesterol, high-density lipoprotein cholesterol and triglyceride concentrations were determined using standard enzymatic automated methods. In patients whose triglyceride levels were less than $400 \mathrm{mg} / \mathrm{dL}$, low-density lipoprotein cholesterol concentrations were estimated using the Friedewald formula. Inflammatory and metabolic markers (adiponectin, adipsin, C-peptide, ghrelin, glucagon-like peptide-1 (GLP-1), glucose-dependent insulinotropic polypeptide, IL-6, leptin, plasminogen activator inhibitor-1 (PAI-1), resistin, tumor necrosis factor (TNF) and visfatin were determined in plasma using the Bio-Plex cytokine assay (Bio-Rad Laboratories Inc., Hercules, CA, USA) according to manufacturer's instructions.

\section{Statistical analysis}

Mean (SD) or percentages (\%) were used to describe the participant's baseline characteristics. Inflammatory and metabolic risk markers of insulin resistance and diabetes were logarithmically transformed to achieve a normal distribution, and the geometric mean and 95\% confidence interval were used to describe these variables. For cross-sectional associations, we used multivariable linear regression models to assess the associations between metabolic risk markers (dependent variables in each model) and dietary vitamin $\mathrm{K}$ intake (independent variable in all models) adjusted for potential confounding variables [age, sex, body mass index, smoking (never, current, past), physical activity (kcal/d), type 2 diabetes mellitus (T2DM), total energy $(\mathrm{kcal} / \mathrm{d}$ ) and fibre intake $(\mathrm{g} / \mathrm{d})$, dietary polyunsaturated fatty acids (PUFA) intake (g/d) and adherence to MedDiet (14-item score, quantitative)]. The selection of potential confounders was done using clinical plausible and bibliographical criteria.
Interaction tests for sex and T2DM (sex"vitamin $\mathrm{K}$ intake, T2DM* vitamin $\mathrm{K}$ intake) were not statistically significant. No interaction was observed for intervention group and changes in dietary phylloquinone intake in any outcome (inclusion of intervention group*one-year changes in dietary phylloquinone intake in the regression models). In the longitudinal analyses, subjects were categorized according to tertiles of changes in dietary vitamin $\mathrm{k}$ intake from baseline to 1-year follow-up. A multivariable linear regression model was fitted to evaluate the relationship between metabolic risk markers at 1-year of follow-up (dependent variables in each model) and tertiles of change in vitamin $\mathrm{k}$ intake (independent variable in all models) adjusting by age, sex, smoking (never, current, past), physical activity (kcal/d), T2DM, intervention group, baseline values of each metabolic marker and changes in BMI, total energy intake $(\mathrm{kcal} / \mathrm{d})$, fiber intake $(\mathrm{g} / \mathrm{d})$, dietary PUFA intake $(\mathrm{g} / \mathrm{d})$ and adherence to MedDiet (the relative change in the 14-item score was expressed as a percentage). All statistical tests were two-tailed, and the significance level was $\mathrm{p}<0.05$. Statistical analysis was performed using SPSS 17.0 for Windows (SPSS Inc, Chicago, IL).

\section{Results}

Of the 568 subjects consecutively recruited, 57 were excluded because they were using anti-inflammatory medication at baseline and 1 because he hd not completed the FFQ at follow-up. Table 1 summarizes the baseline characteristics of the study participants. Study subjects were $67.2 \pm 6.0$ years old and $44.4 \%$ of them were male. Most of them were overweight or obese (92.2\%), had hypertension (91.8\%), were hypercholesterolemic (62.9\%), and 55\% had T2DM. Table 2 presents the baseline and 1-year dietary changes by tertiles of change in dietary phylloquinone intake. Subjects in the highest tertile of change consumed less amounts of vitamin K1 at baseline. This change after intervention was due to a higher consumption of total vegetables and, especially, leafy green vegetables, the primary dietary source of vitamin K1. In cross-sectional analyses at baseline, a negative significant association was found between dietary phylloquinone intake and PAI-1 plasma concentrations, even after adjusting for potential confounders (Table 3), but not for the rest of the metabolic risk markers. However, after 1-year of follow-up, those subjects in the upper tertile of changes in dietary phylloquinone intake showed a significant greater reduction in ghrelin (15.0\%), GIP (12.9\%), GLP-1 (17.6\%), IL-6 (27.9\%), leptin (10.3\%), TNF (26.9\%) and visfatin (24.9\%) than those subjects in the lowest tertile (Table 4). No significant associations were found between changes in dietary phylloquinone intake and other metabolic markers of inflammation, insulin resistance and diabetes. 
Table 1 Baseline characteristics of study subjects by tertiles of change in dietary phylloquinone intake ( $\mu \mathrm{g} / \mathrm{day}$ )

\begin{tabular}{|c|c|c|c|c|}
\hline \multirow[t]{3}{*}{ Characteristics } & \multicolumn{3}{|c|}{ All subjects $(n=510)$} & \multirow[t]{3}{*}{$\mathbf{P}$} \\
\hline & Tertile 1 & Tertile 2 & Tertile 3 & \\
\hline & $(-718.2$ to -69.9$)$ & $(-26.8$ to 69.4$)$ & (70.5 to 767.5 ) & \\
\hline \multicolumn{5}{|l|}{ Clinical characteristics } \\
\hline Men/women, $\mathbf{n}$ & $80 / 92$ & $68 / 107$ & $78 / 85$ & 0.194 \\
\hline Age, years & $66.8 \pm 6.1$ & $68.1 \pm 6.2$ & $66.6 \pm 5.7$ & 0.030 \\
\hline BMI, $\mathrm{kg} / \mathrm{m}^{2}$ & $29.39 \pm 3.17$ & $29.21 \pm 3.18$ & $29.17 \pm 3.05$ & 0.608 \\
\hline Waist circumference, cm & $100.6 \pm 8.6$ & $100.5 \pm 9.2$ & $100.9 \pm 8.9$ & 0.940 \\
\hline Current smoker, n (\%) & $26(15.1)$ & $29(16.6)$ & $16(9.8)$ & 0.561 \\
\hline Type 2 diabetes, $\mathrm{n}(\%)$, & $95(55.2)$ & $100(57.1)$ & $85(52.1)$ & 0.650 \\
\hline Overweight/Obesity, n (\%) & $159(92.4)$ & $162(92.6)$ & $149(91.4)$ & 0.911 \\
\hline Hypertension, n (\%) & $156(90.7)$ & $164(93.7)$ & $148(90.8)$ & 0.121 \\
\hline Dyslipydemia, n (\%) & $103(61.7)$ & $116(68.6)$ & $92(57.9)$ & 0.511 \\
\hline Intervention group $\mathrm{MD}+\mathrm{VOO} / \mathrm{MD}+$ nuts/CD, $\mathrm{n}$ & $44 / 54 / 74$ & $64 / 57 / 54$ & $76 / 45 / 42$ & 0.001 \\
\hline Leisure-time physical activity, METS-min/day & $277.8 \pm 261.9$ & $263.0 \pm 247.8$ & $294.2 \pm 279.4$ & 0.313 \\
\hline \multicolumn{5}{|l|}{ Metabolic risk markers } \\
\hline C-Peptide (ng/mL) & $1.41(1.34$ to 1.49$)$ & $1.33(1.24$ to 1.44$)$ & $1.34(1.25$ to 1.42$)$ & 0.374 \\
\hline Ghrelin (pg/mL) & 12.88 (11.95 to 3.89$)$ & 13.38 (12.31 to 14.54$)$ & $12.77(11.75$ to 13.87$)$ & 0.685 \\
\hline $\mathrm{GIP}(\mathrm{pg} / \mathrm{mL})$ & 92.55 (84.76 to 101.05$)$ & $98.40(89.70$ to 107.95$)$ & $85.72(78.25$ to 93.91$)$ & 0.107 \\
\hline GLP-1 (ng/mL) & $1.22(1.09$ to 1.36$)$ & $1.31(1.17$ to 1.48$)$ & $1.16(1.03$ to 1.30$)$ & 0.312 \\
\hline IL-6 (pg/ml) & 9.96 (8.80 to 11.27$)$ & $10.88(9.63$ to 12.29$)$ & $8.99(7.93$ to 10.19$)$ & 0.102 \\
\hline Leptin (ng/mL) & $2.87(2.60$ to 3.17$)$ & 3.20 (2.88 to 3.57$)$ & $2.91(2.63$ to 3.22$)$ & 0.262 \\
\hline PAI-1 (ng/mL) & 3.20 (3.05 to 3.37$)$ & 3.28 (3.11 to 3.46$)$ & 3.37 (3.21 to 3.54 ) & 0.378 \\
\hline Resistin (ng/mL) & $0.98(0.92$ to 1.05$)$ & 1.05 (0.98 to 1.12$)$ & 1.01 (0.94 to 1.08$)$ & 0.413 \\
\hline TNF (pg/mL) & 13.36 (11.57 to 15.44$)$ & 13.76 (11.89 to 15.93$)$ & 11.35 (9.80 to 13.14$)$ & 0.145 \\
\hline Visfatin (ng/mL) & 4.13 (3.53 to 4.84 ) & 4.54 (3.85 to 5.37 ) & 3.70 (3.13 to 4.37 ) & 0.225 \\
\hline Adiponectin $(\mu \mathrm{g} / \mathrm{mL})$ & 48.87 (42.67 to 55.98) & 49.51 (42.68 to 57.43 ) & 42.37 (35.45 to 50.64$)$ & 0.302 \\
\hline Adipsin $(\mu \mathrm{g} / \mathrm{mL})$ & $1.12(1.01$ to 1.24$)$ & $1.14(1.03$ to 1.28$)$ & 0.91 (0.77 to 1.08$)$ & 0.029 \\
\hline
\end{tabular}

\section{Discussion}

The results of this study show, for the first time, that an increased dietary intake of phylloquinone is associated with an improvement in inflammatory and other metabolic risk markers related to insulin resistance and diabetes, thus supporting a protective role of vitamin $\mathrm{K}$ on low-grade chronic inflammatory diseases.

In recent years, vitamin $\mathrm{K}$ has been attributed a putative role in glucose metabolism, insulin resistance and T2DM [17]. Recently, in a prospective study our group has shown that a higher dietary phylloquinone intake was associated to a lower risk of developing T2DM. However, the exact mechanisms underlying this relationship remain still unknown. One potential explanatory mechanism could be related to the role of vitamin $\mathrm{K}$ as a cofactor in the carboxylation of vitamin K-dependent proteins, such as osteocalcin [18], GAS6 or Protein S [19] and through their role on NF-kB [20-22].
Additionally, some authors have suggested that inflammation may be modulated by a possible antioxidant effect of vitamin K [23].

A cross-sectional study conducted in a subsample from the Framingham Offspring Study, showed an inverse association between 2-fold changes in usual dietary phylloquinone intake or plasma phylloquinone concentrations and seven of the fourteen peripheral inflammatory markers measured [9]. However, the same authors, in a cross-sectional analysis conducted on 400 healthy elderly men and women, did not find any significant association between plasma phylloquinone concentrations and C-reactive protein or IL-6 after adjusting for potential confounders [10]. Similar to the results from Shea and co-workers, we also failed to find any significant association between dietary phylloquinone intake and most of the metabolic risk markers analysed. However, our longitudinal findings are generally consistent with in- 
Table 2 Baseline and 1-year change of dietary characteristics by tertiles of change in dietary phylloquinone intake ( $\mu \mathrm{g} / \mathrm{day})$

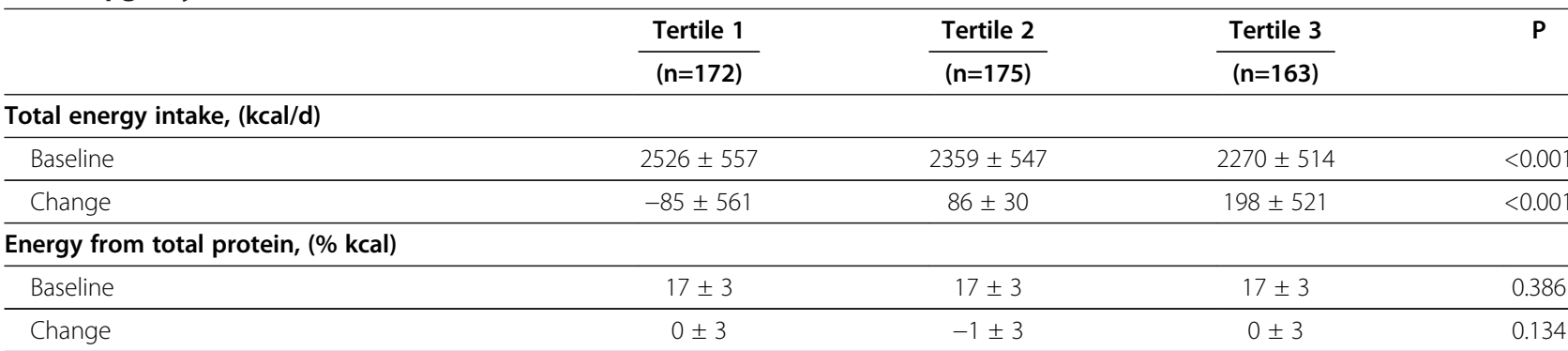

Energy from total carbohidrates, (\% kcal)

\begin{tabular}{lllll}
\hline Baseline & $42 \pm 6$ & $42 \pm 7$ & $42 \pm 7$ & 0.982 \\
\hline Change & $-2 \pm 6$ & $-2 \pm 7$ & $-2 \pm 8$ & 0.958 \\
\hline
\end{tabular}

\section{Fiber intake, $\mathrm{g} / 1,000 \mathrm{kcal}$}

\begin{tabular}{|c|c|c|c|c|}
\hline Baseline & $12 \pm 4$ & $12 \pm 3$ & $11 \pm 3$ & 0.023 \\
\hline Change & $-1 \pm 3$ & $0 \pm 2$ & $2 \pm 3$ & $<0.001$ \\
\hline \multicolumn{5}{|c|}{ Energy from total fat, (\% kcal) } \\
\hline Baseline & $39 \pm 6$ & $39 \pm 6$ & $39 \pm 6$ & 0.393 \\
\hline Change & $2 \pm 6$ & $3 \pm 7$ & $2 \pm 8$ & 0.240 \\
\hline
\end{tabular}

Saturated fatty acids, (\%)

\begin{tabular}{|c|c|c|c|c|}
\hline Baseline & $27 \pm 4$ & $26 \pm 5$ & $26 \pm 4$ & 0.829 \\
\hline Change & $-2 \pm 5$ & $-3 \pm 5$ & $-2 \pm 5$ & 0.271 \\
\hline \multicolumn{5}{|l|}{ MUFA, (\%) } \\
\hline Baseline & $49 \pm 5$ & $48 \pm 5$ & $49 \pm 5$ & 0.242 \\
\hline Change & $1 \pm 6$ & $2 \pm 5$ & $1 \pm 6$ & 0.140 \\
\hline \multicolumn{5}{|l|}{ PUFA, (\%) } \\
\hline Baseline & $17 \pm 4$ & $17 \pm 4$ & $16 \pm 4$ & 0.456 \\
\hline Change & $0 \pm 5$ & $1 \pm 5$ & $1 \pm 5$ & 0.221 \\
\hline
\end{tabular}

Phylloquinone intake, $(\mu \mathrm{g} / \mathrm{d})$

\begin{tabular}{|c|c|c|c|c|}
\hline Baseline & $490 \pm 229$ & $401 \pm 209$ & $297 \pm 163$ & $<0.001$ \\
\hline Change & $-176 \pm 150$ & $16 \pm 25$ & $266 \pm 164$ & $<0.001$ \\
\hline \multicolumn{5}{|c|}{ Vegetable consumption, $(\mathrm{g} / \mathrm{d})$} \\
\hline Baseline & $413 \pm 167$ & $380 \pm 182$ & $335 \pm 186$ & $<0.001$ \\
\hline Change & $-42 \pm 180$ & $17 \pm 131$ & $141 \pm 175$ & $<0.001$ \\
\hline \multicolumn{5}{|c|}{ Leafy green vegetables, (g/d) } \\
\hline Baseline & $112 \pm 47$ & $95 \pm 47$ & $75 \pm 44$ & $<0.001$ \\
\hline Change & $-30 \pm 38$ & $1 \pm 20$ & $55 \pm 44$ & $<0.001$ \\
\hline \multicolumn{5}{|c|}{ Other vegetables, $(\mathrm{g} / \mathrm{d})$} \\
\hline Baseline & $276 \pm 138$ & $265 \pm 142$ & $239 \pm 147$ & 0.053 \\
\hline Change & $-13 \pm 149$ & $7 \pm 120$ & $79 \pm 153$ & $<0.001$ \\
\hline \multicolumn{5}{|c|}{ Fruit consumption, $(\mathrm{g} / \mathrm{d})$} \\
\hline Baseline & $479 \pm 258$ & $451 \pm 240$ & $432 \pm 251$ & 0.221 \\
\hline Change & $-12 \pm 223$ & $36 \pm 211$ & $67 \pm 238$ & 0.005 \\
\hline \multicolumn{5}{|c|}{ Legume consumption, $(\mathrm{g} / \mathrm{d})$} \\
\hline Baseline & $20 \pm 11$ & $19 \pm 12$ & $17 \pm 9$ & 0.046 \\
\hline Change & $1 \pm 12$ & $4 \pm 12$ & $7 \pm 13$ & $<0.001$ \\
\hline \multicolumn{5}{|c|}{ Cereal consumption, $(\mathrm{g} / \mathrm{d})$} \\
\hline Baseline & $270 \pm 103$ & $256 \pm 102$ & $249 \pm 103$ & 0.150 \\
\hline Change & $-27 \pm 100$ & $-10 \pm 119$ & $-1 \pm 113$ & 0.091 \\
\hline
\end{tabular}


Table 2 Baseline and 1-year change of dietary characteristics by tertiles of change in dietary phylloquinone intake ( $\mu \mathrm{g} /$ day) (Continued)

\begin{tabular}{|c|c|c|c|c|}
\hline \multicolumn{5}{|c|}{ Dairy product consumption, $(\mathrm{g} / \mathrm{d})$} \\
\hline Baseline & $357 \pm 227$ & $371 \pm 252$ & $349 \pm 195$ & 0.656 \\
\hline Change & $-8 \pm 212$ & $-8 \pm 190$ & $2 \pm 181$ & 0.854 \\
\hline \multicolumn{5}{|c|}{ Meat consumption, (g/d) } \\
\hline Baseline & $154 \pm 61$ & $148 \pm 61$ & $143 \pm 56$ & 0.243 \\
\hline Change & $-14 \pm 58$ & $-12 \pm 62$ & $-2 \pm 61$ & 0.137 \\
\hline \multicolumn{5}{|c|}{ Fish consumption, (g/d) } \\
\hline Baseline & $115 \pm 46$ & $106 \pm 46$ & $106 \pm 42$ & 0.099 \\
\hline Change & $-1 \pm 52$ & $9 \pm 46$ & $15 \pm 43$ & 0.008 \\
\hline \multicolumn{5}{|c|}{ Alcohol intake, (g/d) } \\
\hline Baseline & $13 \pm 19$ & $10 \pm 19$ & $7 \pm 12$ & 0.013 \\
\hline Change & $0 \pm 13$ & $-1 \pm 17$ & $1 \pm 9$ & 0.173 \\
\hline \multicolumn{5}{|c|}{ Olive oil consumption, (g/d) } \\
\hline Baseline & $39 \pm 15$ & $36 \pm 14$ & $38 \pm 15$ & 0.248 \\
\hline Change & $5 \pm 19$ & $11 \pm 19$ & $9 \pm 20$ & 0.021 \\
\hline \multicolumn{5}{|c|}{ Nut consumption, (g/d) } \\
\hline Baseline & $16 \pm 17$ & $14 \pm 15$ & $11 \pm 13$ & 0.029 \\
\hline Change & $7 \pm 25$ & $10 \pm 25$ & $11 \pm 24$ & 0.185 \\
\hline \multicolumn{5}{|c|}{ 14-item PREDIMED MedDiet Score } \\
\hline Baseline & $9 \pm 2$ & $9 \pm 2$ & $8 \pm 2$ & 0.443 \\
\hline Change & $1 \pm 2$ & $1 \pm 2$ & $2 \pm 2$ & 0.031 \\
\hline
\end{tabular}

vitro studies or those of a previous epidemiological study. In-vitro studies found that the production of the proinflammatory cytokines IL- 6 or TNF by human gingival fibroblast or mouse macrophage cells, respectively, decreased when cells were incubated with different vitamin K family compounds [21,24]. Other studies have found that vitamin $\mathrm{K}$ suppresses inflammation by lowering the expression of genes for some proinflammatory cytokines, such as IL- 6 , IL- $1 \beta$ and TNF $[8,22]$. However, only a single 3 -year, double-blind, randomized controlled trial has been conducted to evaluate the effect of vitamin $\mathrm{K}$ supplementation on peripheral inflammatory marker concentrations. In that study, conducted with 379 healthy men and women, no significant relationship between plasma phylloquinone levels and inflammation markers was shown [10]. In our study we observed a significant improvement in the inflammatory status (leptin, IL-6, TNF) and a decrease in other metabolic risk markers related to insulin resistance and diabetes such as visfatin, ghrelin, GIP and GLP-1 among subjects who increased their dietary intake of phylloquinone after 1-year follow-up, thus contributing to extend the knowledge on the role of vitamin $\mathrm{K}$ in humans and to support our previous results on dietary phylloquinone intake and T2DM incidence [7]. The discrepancies between our results and those previously published could be partly explained by the differences between the populations. Our participants were elderly subjects at high cardiovascular risk, whereas, subjects in the study of Shea [9] were healthy and generally free of chronic disease. This may contribute to explain why inflammatory cytokines remained unchanged in that study. Moreover, in our study we assessed the association of inflammation with dietary phylloquinone intake instead of plasma phylloquinone as was done in the previous study. Whether circulating levels of phylloquinone are correlated to dietary phylloquinone intake remains to be elucidated.

It must be noted that leptin, IL6 and TNF are proinflammatory cytokines with a recognised role in the development and progression of insulin resistance, T2DM and cardiovascular disease [25,26]. Also visfatin and ghrelin appears as important mediators of inflammation in addition to glucose-lowering and insulin-mimicking/ sensitizing effects or a suppressive role of ghrelin in the release of insulin from the pancreatic islets [27-30]. A strong inverse correlation between plasma ghrelin concentrations and insulin resistance has been observed in several studies [30-32] and lower concentrations of ghrelin have been observed in T2DM subjects [33]. The negative relationship between changes in dietary phylloquinone intake and plasma incretin concentrations 
Table 3 Cross-sectional associations between intake of $100 \mu \mathrm{g}$ of dietary phylloquinone and inflammatory or metabolic markers at baseline

Change in inflammatory marker for 100 additional $\mu \mathrm{g}$ of dietary phylloquinone intake

(95\% confidence intervals)

\begin{tabular}{lll}
\hline C-Peptide $(\mathbf{n g} / \mathbf{m L})$ & $-0.73(-2.68$ to 1.27$)$ & 0.472 \\
\hline Ghrelin $(\mathbf{p g} / \mathbf{m L})$ & $-1.25(-3.76$ to 1.33$)$ & 0.339 \\
\hline GIP $(\mathbf{p g} / \mathbf{m L})$ & $-0.13(-2.98$ to 2.80$)$ & 0.928 \\
\hline GLP-1 $\mathbf{n g} / \mathbf{m L})$ & $-1.81(-5.40$ to 1.91$)$ & 0.335 \\
\hline IL-6 $(\mathbf{p g} / \mathbf{m l})$ & $-1.68(-5.46$ to 2.25$)$ & 0.396 \\
\hline Leptin $(\mathbf{n g} / \mathbf{m L})$ & $-1.48(-3.80$ to 0.90$)$ & 0.221 \\
\hline PAI-1 $\mathbf{n g} / \mathbf{m L})$ & $-1.64(-3.23$ to -0.0$) 2$ & 0.047 \\
\hline Resistin $(\mathbf{n g} / \mathbf{m L})$ & $-1.42(-3.61$ to 0.81$)$ & 0.210 \\
\hline TNF $(\mathbf{p g} / \mathbf{m L})$ & $-1.24(-5.73$ to 3.46$)$ & 0.598 \\
\hline Visfatin $(\mathbf{n g} / \mathbf{m L})$ & $-1.42(-6.54$ to 3.98) & 0.599 \\
\hline Adiponectin $(\boldsymbol{\mu g} / \mathbf{m L})$ & $2.45(-2.23$ to 7.35$)$ & 0.310 \\
\hline Adipsin $(\boldsymbol{\mu g} / \mathbf{m L})$ & $1.21(-2.84$ to 5.43$)$ & 0.563 \\
\hline
\end{tabular}

Multivariable linear regression models were used for analysis. Data are given in \% of change (95\% IC). Models are adjusted for sex, age, body-mass-index, smoking (never, current, past), total energy intake (kcal/d), dietary fibre intake (g/d), dietary polyunsaturated fatty acids intake (g/d), physical activity (kcal/d), Mediterranean Diet Score (14-item PREDIMED score, quantitative), and T2DM. GIP Gastric inhibitory polypeptide, GLP-1 Glucagon-like peptide-1, IL-6 Interleukin-6, PAI-1

Plasminogen activator inhibitor-1, TNF Tumor Necrosis Factor a.

observed in our study could be explained because a higher intake of phylloquinone may promote better glycemic control thus leading less necessary the glucose and insulin regulation mediated by incretins. However, the pleiotropic role recently attributed to incretins could also contribute to explain our results. Although there is growing evidence that incretin hormones (GIP and GLP-1) simulate glucose-dependent insulin secretion and stimulate pancreatic synthesis of insulin, a novel link between inflammation and incretin hormones has been proposed. First, IL-6 increase GLP-1 production in intestinal L cells and alpha pancreatic cells improving insulin secretion and glycemia [34]. Therefore, the reduction of
IL-6 levels observed in the subjects allocated in the highest tertile of change in phylloquinone intake could partly explain the reduction in GLP-1 concentrations in this group. Nie et al., have also demonstrated the capacity of GIP to activate inflammatory response and promote secretion of pro-inflammatory cytokines and chemokines in cell culture adipocytes [35]. Additionally, a potential role of GIP on adipose tissue insulin resistance mediated by osteopontin regulation has also been suggested [36]. The results from our study could be related to the potential role of incretins on adipose tissue in a fasting situation rather than to their established role on pancreatic cells in a post-prandial state. Whether or not GIP

Table 4 Longitudinal associations between one-year changes in inflammatory or metabolic markers and tertiles of change in dietary phylloquinone intake ( $\mu \mathrm{g} /$ day)

\begin{tabular}{|c|c|c|c|c|c|c|}
\hline & Tertile 1 & Tertile 2 & $\mathbf{p}$ & Tertile 3 & $\mathrm{p}$ & $\mathrm{p}$ for trend \\
\hline C-Peptide (ng/mL) & Ref. & $-2.19(-8.35$ to 4.40$)$ & 0.505 & $-6.10(-12.61$ to 0.91$)$ & 0.086 & 0.083 \\
\hline Ghrelin (pg/mL) & Ref. & $1.69(-8.58$ to 13.11$)$ & 0.758 & $-15.00(-24.49$ to -4.32$)$ & 0.007 & 0.003 \\
\hline GIP (pg/mL) & Ref. & 0.08 (-10.17 to 11.49$)$ & 0.989 & $-12.94(-22.75$ to -1.89$)$ & 0.023 & 0.014 \\
\hline GLP-1 (ng/mL) & Ref. & $0.21(-12.99$ to 15.42$)$ & 0.977 & $-17.64(-29.67$ to -3.56$)$ & 0.016 & 0.009 \\
\hline IL-6 (pg/ml) & Ref. & $-4.50(-18.17$ to 11.46$)$ & 0.559 & $-27.89(-39.20$ to -14.48$)$ & $<0.001$ & $<0.001$ \\
\hline Leptin $(\mathrm{ng} / \mathrm{mL})$ & Ref. & $-7.79(-15.61$ to 0.76$)$ & 0.073 & $-10.26(-18.62$ to -1.03$)$ & 0.030 & 0.044 \\
\hline PAl-1 (ng/mL) & Ref. & $-3.06(-10.65$ to 5.17$)$ & 0.454 & $-3.24(-11.61$ to 5.93$)$ & 0.476 & 0.529 \\
\hline Resistin (ng/mL) & Ref. & $-2.43(-11.03$ to 7.01$)$ & 0.601 & $-6.66(-15.71$ to 3.35$)$ & 0.184 & 0.180 \\
\hline TNF (pg/mL) & Ref. & $-3.96(-20.47$ to 15.98$)$ & 0.674 & $-26.89(-40.65$ to -9.94$)$ & 0.003 & 0.002 \\
\hline Visfatin $(\mathrm{ng} / \mathrm{mL})$ & Ref. & $-8.08(-24.73$ to 12.24$)$ & 0.408 & $-24.90(-39.86$ to -6.22$)$ & 0.012 & 0.010 \\
\hline Adiponectin $(\mu \mathrm{g} / \mathrm{mL})$ & Ref. & $-3.27(-19.20$ to 15.79$)$ & 0.716 & $-7.34(-23.98$ to 12.93$)$ & 0.449 & 0.452 \\
\hline Adipsin $(\mu \mathrm{g} / \mathrm{mL})$ & Ref. & $-2.45(-15.37$ to 12.45$)$ & 0.732 & $-7.39(-20.90$ to 8.44$)$ & 0.340 & 0.332 \\
\hline
\end{tabular}

Multivariable linear regression models were used for analysis. Data are given in \% of change (95\% IC). Models are adjusted for sex, age, change of

body-mass-index, smoking (never, current, past), change of total energy intake (kcal/d), change of fibre intake ( $\mathrm{g} / \mathrm{d}$ ), change of dietary polyunsaturated fatty acids intake (g/d), physical activity (kcal/d), intervention group, change of Mediterranean Diet Score (\%), T2DM and baseline values of each metabolic marker. GIP Gastric inhibitory polypeptide, GLP-1 Glucagon-like peptide-1, IL-6 Interleukin-6, PAI-1 Plasminogen activator inhibitor-1, TNF, Tumor Necrosis Factor a 
and GLP-1 need to be considered as new adipokynes or related pro-inflammatory markers could not be elucitaded from the results of our study. Specific studies are needed to get a deeper understanding of the exact role of incretins on adipose tissue and their interaction with the rest of adipokynes.

Our study has several limitations. It should be kept in mind that the subjects in our study were randomly allocated to a healthy Mediterranean diet that could partially account for the reduction in peripheral metabolic risk markers in some of them although no significant interaction between intervention group and dietary phylloquinone intake was observed for any of the outcomes. In order to minimize the potential effect of a healthy dietary pattern on inflammatory response we have adjusted the regression models for the adherence to a MedDiet. Subjects in the PREDIMED study reported a higher dietary intake of phylloquinone than in other epidemiological studies, probably because this study was conducted in a Mediterranean country where the consumption of fruit and vegetables is high. In populations with a lower consumption of phylloquinone or poor nutrition an increase in dietary vitamin K1 would probably be much more beneficial. The cohort studied was elderly and at high risk of cardiovascular disease, so our findings cannot be generalized to younger or healthier individuals. Because there is no perfect correlation between dietary phylloquinone intake and its absorption it would be interesting in the future to evaluate the associations showed in our study not only with dietary phylloquinone intake but also with a circulating marker of vitamin $\mathrm{K}$ status. Finally, we cannot discount a slight overestimation of dietary phylloquinone intake due to the use of FFQ and the USDA Food Database Composition. Although the FFQ used in our study was not specifically validated for phylloquinone intake, the intraclass correlation coefficient of vegetables (the main source of dietary vitamin K1) was 0.81 , one of the highest coefficients obtained during the validation of the FFQ in the PREDIMED cohort [13]. Balanced against these limitations, the main strength of our study is its longitudinal design, which enables us to suggest a cause-effect relationship between changes in dietary phylloquinone intake and changes in inflammatory and related metabolic risk markers. In addition, our study was conducted in a large sample of individuals, and measured a panel of adipokines and related molecules involved in inflammation, glucose metabolism and cardiovascular risk.

In summary, our results support that an increase in dietary phylloquinone intake can lead to an improvement in inflammation and inflammatory-related molecules and also support the contention that high vitamin K1 intake has a beneficial effect on cardiovascular disease and other inflammation-related disorders.

\section{Abbreviations}

FFQ: Food Frequency Questionnaire; GLP-1: Glucagon-like peptide 1; IL: Interleukin; MedDiet: Mediterranean Diet; PAl-1: Plasminogen activator inhibitor-1; T2DM: Type 2 diabetes mellitus; TNF: Tumor necrosis factor alpha.

\section{Competing interests}

The authors declare that they have no competing interests.

\section{Authors' contributions}

JS, RE, MAM and MB contributed to the study design, study performance, data analysis and writing of the manuscript; MJ-F and RC performed biochemical measurements and contributed to the data analysis and the writing of the manuscript. MP and JM revised the manuscript critically for important intellectual content. All authors had a substantial input in critically appraising the manuscript and approved the final version to be published. All authors read and approved the final manuscript.

\section{Acknowledgments}

We thank all the participants of the PREDIMED study for their enthusiastic collaboration, the PREDIMED personnel for excellent assistance and the personnel of all affiliated primary care centers. CIBERobn and RTIC RD 06/ 0045 are initiatives of ISCIII, Spain. We also acknowledge the grants from Centro Nacional de Investigaciones Cardiovasculares CNIC 06/2007, Fondo de Investigación Sanitaria PI 07/0473, Ministerio de Ciencia e Innovación (AGL2009-13906-C02, AGL2010-22319-C03), and Fundación Mapfre 2010, Government of the Basque Country (IT386-10), University of the Basque Country (UFI 11/32).

\section{Author details}

${ }^{1}$ Human Nutrition Unit, Faculty of Medicine and Health Sciences, IISPV, Universitat Rovira i Virgili, C/Sant Llorenç 21, 43201, Reus, Spain. ${ }^{2}$ CIBERobn Physiopathology of Obesity and Nutrition, Institute of Health Carlos III (ISCIII), Madrid, Spain. ${ }^{3}$ PREDIMED Network (RD 06/0045), ISCIII, Madrid, Spain.

${ }^{4}$ Department of Internal Medicine, Institut d'Investigacions Biomèdiques August Pi Sunyer (IDIBAPS), Hospital Clínic, Barcelona, Spain. ${ }^{5}$ Department of Nutrition and Food Science, University of Pais Vasco, Vitoria, Spain.

${ }^{6}$ Department of Preventive Medicine and Public Health, University of Navarra, Pamplona, Spain.

Received: 21 November 2012 Accepted: 5 January 2013 Published: 8 January 2013

\section{References}

1. Iwamoto J, Sato Y, Takeda T, Matsumoto H: Bone quality and vitamin K2 in type 2 diabetes: review of preclinical and clinical studies. Nutr Rev 2011, 69(3):162-167.

2. Shearer MJ: The roles of vitamins $D$ and $K$ in bone health and osteoporosis prevention. Proc Nutr Soc 1997, 56(3):915-937.

3. Booth SL: Roles for vitamin K beyond coagulation. Annu Rev Nutr 2009, 29:89-110.

4. Yoshida M, Booth SL, Meigs JB, Saltzman E, Jacques PF: Phylloquinone intake, insulin sensitivity, and glycemic status in men and women. Am J Clin Nutr 2008, 88(1):210-215.

5. Kumar R, Binkley N, Vella A: Effect of phylloquinone supplementation on glucose homeostasis in humans. Am J Clin Nutr 2010, 92(6):1528-1532.

6. Beulens JW, van Der ADL, Grobbee DE, Sluijs I, Spijkerman AM, van der Schouw YT: Dietary phylloquinone and menaquinones intakes and risk of type 2 diabetes. Diabetes Care 2010, 33(8):1699-1705.

7. Ibarrola-Jurado N, Salas-Salvado J, Martinez-Gonzalez MA, Bullo M: Dietary phylloquinone intake and risk of type 2 diabetes in elderly subjects at high risk of cardiovascular disease. Am J Clin Nutr 2012, 96(5):1113-1118.

8. Ohsaki Y, Shirakawa H, Hiwatashi K, Furukawa Y, Mizutani T, Komai M: Vitamin K suppresses lipopolysaccharide-induced inflammation in the rat. Biosci Biotechnol Biochem 2006, 70(4):926-932.

9. Shea MK, Booth SL, Massaro JM, Jacques PF, D'Agostino RBS, DawsonHughes B, Ordovas JM, O'Donnell CJ, Kathiresan S, Keaney JF Jr, Vasan RS, Benjamin EJ: Vitamin K and vitamin D status: associations with inflammatory markers in the Framingham Offspring Study. Am J Epidemiol 2008, 167(3):313-320.

10. Shea MK, Dallal GE, Dawson-Hughes B, Ordovas JM, O'Donnell CJ, Gundberg CM, Peterson JW, Booth SL: Vitamin K, circulating cytokines, and bone 
mineral density in older men and women. Am J Clin Nutr 2008, 88(2):356-363.

11. Estruch R, Martinez-Gonzalez MA, Corella D, Salas-Salvado J, Ruiz-Gutierrez V, Covas MI, Fiol M, Gomez-Gracia E, Lopez-Sabater MC, Vinyoles E, Aros F, Conde M, Lahoz C, Lapetra J, Saez G, Ros E, PREDIMED Study Investigators: Effects of a Mediterranean-style diet on cardiovascular risk factors: a randomized trial. Ann Intern Med 2006, 145(1):1-11.

12. Martinez-Gonzalez MA, Corella D, Salas-Salvado J, Ros E, Covas MI, Fiol M Warnberg J, Aros F, Ruiz-Gutierrez V, Lamuela-Raventos RM, Lapetra J, Munoz MA, Martinez JA, Saez G, Serra-Majem L, Pinto X, Mitjavila MT, Tur JA, Portillo MD, Estruch R, for the PREDIMED Study Investigators: Cohort profile: design and methods of the PREDIMED study. Int J Epidemiol 2012, 41(2):377-385.

13. Fernandez-Ballart JD, Pinol JL, Zazpe I, Corella D, Carrasco P, Toledo E, PerezBauer M, Martinez-Gonzalez MA, Salas-Salvado J, Martin-Moreno JM: Relative validity of a semi-quantitative food-frequency questionnaire in an elderly Mediterranean population of Spain. Br J Nutr 2010, 103(12):1808-1816.

14. Schroder H, Fito M, Estruch R, Martinez-Gonzalez MA, Corella D, SalasSalvado J, Lamuela-Raventos R, Ros E, Salaverria I, Fiol M, Lapetra J, Vinyoles E, Gomez-Gracia E, Lahoz C, Serra-Majem L, Pinto X, Ruiz-Gutierrez V, Covas Ml: A short screener is valid for assessing Mediterranean diet adherence among older Spanish men and women. J Nutr 2011, 141(6):1140-1145.

15. Moreiras O, Carbajal A, Cabrera L, Cuadrado C: Tablas de composición de los alimentos. (Food Composition Tables). 9th edition. Madrid: Pirámide; 2005.

16. Mataix Verdú J: Tabla de composicion de alimentos [Food Composition Tables]. 4th edition. Granada (Spain): Universidad de Granada; 2003.

17. Pan $Y$, Jackson RT: Dietary phylloquinone intakes and metabolic syndrome in US young adults. J Am Coll Nutr 2009, 28(4):369-379.

18. Bullo M, Moreno-Navarrete JM, Fernandez-Real JM, Salas-Salvado J: Total and undercarboxylated osteocalcin predict changes in insulin sensitivity and beta cell function in elderly men at high cardiovascular risk. Am J Clin Nutr 2012, 95(1):249-255.

19. Fernandez-Fernandez L, Bellido-Martin L, Garcia de Frutos P: Growth arrestspecific gene 6 (GAS6). An outline of its role in haemostasis and inflammation. Thromb Haemost 2008, 100(4):604-610.

20. Ozaki I, Zhang H, Mizuta T, Ide Y, Eguchi Y, Yasutake T, Sakamaki T, Pestell $\mathrm{RG}$, Yamamoto $\mathrm{K}$ : Menatetrenone, a vitamin $\mathrm{K} 2$ analogue, inhibits hepatocellular carcinoma cell growth by suppressing cyclin D1 expression through inhibition of nuclear factor kappaB activation. Clin Cancer Res 2007, 13(7):2236-2245.

21. Tanaka S, Nishiumi S, Nishida M, Mizushina Y, Kobayashi K, Masuda A, Fujita T, Morita Y, Mizuno S, Kutsumi H, Azuma T, Yoshida M: Vitamin K3 attenuates lipopolysaccharide-induced acute lung injury through inhibition of nuclear factor-kappaB activation. Clin Exp Immunol 2010, 160(2):283-292.

22. Ohsaki Y, Shirakawa H, Miura A, Giriwono PE, Sato S, Ohashi A, Iribe M, Goto $T$, Komai M: Vitamin K suppresses the lipopolysaccharide-induced expression of inflammatory cytokines in cultured macrophage-like cells via the inhibition of the activation of nuclear factor kappaB through the repression of IKKalpha/beta phosphorylation. J Nutr Biochem 2010 21(11):1120-1126.

23. Li J, Lin JC, Wang H, Peterson JW, Furie BC, Furie B, Booth SL, Volpe JJ, Rosenberg PA: Novel role of vitamin $\mathrm{k}$ in preventing oxidative injury to developing oligodendrocytes and neurons. J Neurosci 2003, 23(13):5816-5826.

24. Reddi K, Henderson B, Meghji S, Wilson M, Poole S, Hopper C, Harris M, Hodges SJ: Interleukin 6 production by lipopolysaccharide-stimulated human fibroblasts is potently inhibited by naphthoquinone (vitamin K) compounds. Cytokine 1995, 7(3):287-290.

25. Shoelson SE, Lee J, Goldfine AB: Inflammation and insulin resistance. J Clin Invest 2006, 116(7):1793-1801.

26. Goldberg RB: Cytokine and cytokine-like inflammation markers, endothelial dysfunction, and imbalanced coagulation in development of diabetes and its complications. J Clin Endocrinol Metab 2009, 94(9):3171-3182

27. Kim SR, Bae YH, Bae SK, Choi KS, Yoon KH, Koo TH, Jang HO, Yun I, Kim KW, Kwon YG, Yoo MA, Bae MK: Visfatin enhances ICAM-1 and VCAM-1 expression through ROS-dependent NF-kappaB activation in endothelial cells. Biochim Biophys Acta 2008, 1783(5):886-895.
28. Lee WJ, Wu CS, Lin H, Lee IT, Wu CM, Tseng JJ, Chou MM, Sheu WH: Visfatin-induced expression of inflammatory mediators in human endothelial cells through the NF-kappaB pathway. Int J Obes (Lond) 2009, 33(4):465-472

29. Tong J, Prigeon RL, Davis HW, Bidlingmaier M, Kahn SE, Cummings DE, Tschop MH, D'Alessio D: Ghrelin suppresses glucose-stimulated insulin secretion and deteriorates glucose tolerance in healthy humans. Diabetes 2010, 59(9):2145-2151.

30. Schofl C, Horn R, Schill T, Schlosser HW, Muller MJ, Brabant G: Circulating ghrelin levels in patients with polycystic ovary syndrome. J Clin Endocrinol Metab 2002, 87(10):4607-4610.

31. Poykko SM, Kellokoski E, Horkko S, Kauma H, Kesaniemi YA, Ukkola O: Low plasma ghrelin is associated with insulin resistance, hypertension, and the prevalence of type 2 diabetes. Diabetes 2003, 52(10):2546-2553.

32. McLaughlin T, Abbasi F, Lamendola C, Frayo RS, Cummings DE: Plasma ghrelin concentrations are decreased in insulin-resistant obese adults relative to equally obese insulin-sensitive controls. J Clin Endocrinol Metab 2004, 89(4):1630-1635.

33. Ostergard T, Hansen TK, Nyholm B, Gravholt CH, Djurhuus CB, Hosoda H, Kangawa K, Schmitz O: Circulating ghrelin concentrations are reduced in healthy offspring of Type 2 diabetic subjects, and are increased in women independent of a family history of Type 2 diabetes. Diabetologia 2003, 46(1):134-136.

34. Ellingsgaard H, Hauselmann I, Schuler B, Habib AM, Baggio LL, Meier DT, Eppler E, Bouzakri K, Wueest S, Muller YD, Hansen AM, Reinecke M, Konrad D, Gassmann M, Reimann F, Halban PA, Gromada J, Drucker DJ, Gribble FM, Ehses JA, Donath MY: Interleukin-6 enhances insulin secretion by increasing glucagon-like peptide- 1 secretion from $L$ cells and alpha cells. Nat Med 2011, 17(11):1481-1489.

35. Nie Y, Ma RC, Chan JC, Xu H, Xu G: Glucose-dependent insulinotropic peptide impairs insulin signaling via inducing adipocyte inflammation in glucose-dependent insulinotropic peptide receptor-overexpressing adipocytes. FASEB J 2012, 26(6):2383-2393.

36. Omar B, Banke E, Guiguis E, Kesson L, Manganiello V, Lyssenko V, Groop L, Gomez MF, Degerman E: Regulation of the pro-inflammatory cytokine osteopontin by GIP in adipocytes - A role for the transcription factor NFAT and phosphodiesterase 3B. Biochem Biophys Res Commun 2012, 425(4):812-819.

doi:10.1186/1475-2840-12-7

Cite this article as: Juanola-Falgarona et al.: Association between dietary phylloquinone intake and peripheral metabolic risk markers related to insulin resistance and diabetes in elderly subjects at high cardiovascular risk. Cardiovascular Diabetology 2013 12:7.

\section{Submit your next manuscript to BioMed Central and take full advantage of:}

- Convenient online submission

- Thorough peer review

- No space constraints or color figure charges

- Immediate publication on acceptance

- Inclusion in PubMed, CAS, Scopus and Google Scholar

- Research which is freely available for redistribution 